\title{
LETTERS
}

\section{An unusual case of Behçet's disease presenting with bilateral internal carotid artery occlusion}

Behçet's disease (BD) is a multisystemic recurrent inflammatory disorder, which is originally described as a triad of oral and genital ulcerations with uveitis. As vasculitis of the vasa vasorum is the main pathological hallmark of $\mathrm{BD}$, it is generally seen in the form of superficial thrombophlebitis or occlusion of major veins; however arterial obstruction and aneurysms may also be seen to a lesser extent. ${ }^{1}$ We present a patient with BD who developed bilateral internal carotid artery (ICA) occlusions.

\section{Case report}

A 43 year old, right handed male patient was referred to Ege University Neurology Department for evaluation of an acute onset righ sided weakness, fever, headache, and difficulty with gait and speech in August 2001

On admission, he was alert and fully oriented. His temperature was $38^{\circ} \mathrm{C}$, pulse was regular $(90 / \mathrm{min})$, blood pressure was $150 / 80$ $\mathrm{mm} \mathrm{Hg}$. His speech was severely dysarthric but he could name, repeat, read, and follow instructions. His cranial nerves and fundoscopic examination were normal. His gait was wide based and unsteady. He had four sided mild weakness, which was prominent on the right. Muscle stretch reflexes were normal but plantar reflexes were extensor bilaterally. Hi coordination was impaired in proportion to weakness in all four extremities. He had mild nuchal rigidity of the neck with positive Brudzinski's sign. On physical examination, erythema nodosum like dark red, painful lesions were noticed on both anterior aspects of the legs. His ophthalmological examination did not reveal any signs of uveitis. He also complained of pain and fever in his scrotum and urological examination showed swelling, induration, and marked tenderness of epididymia on both sides as the clinical findings of epididymitis

His medical history showed that he had complained about recurrent oral aphthous lesions and aforementioned skin lesions for 8 to 10 years without medical consultation. $\mathrm{He}$ had no other medical history associated with $\mathrm{BD}$. He was a moderate cigarette smoker for 20 years.

Laboratory tests were consistent with an inflammatory condition with a high erythrocyte sedimentation rate ( $100 \mathrm{~mm} \mathrm{lst} \mathrm{h)} \mathrm{and} \mathrm{C}$ reactive protein $(12.27 \mathrm{mg} / \mathrm{dl}$; normal range 0-5 mg/dl) levels. CSF examination, serum immunoglobulin levels, platelet count, protein $\mathrm{C}$, protein S, antithrombin III, $\mathrm{C} 3$ and $\mathrm{C} 4$ complement, rheumatoid factor, and lipid levels were within the normal range. Serum antinuclear, antineutrophil cytoplasmic and anticardiolipin antibodies were negative. ECG, 2D echo, chest radiograph, abdominal ultrasonography, and colour Doppler ultrasonography of the lower extremity vessels were normal. Cranial magnetic resonance maging showed diffuse cerebral atrophy and chronic ischaemic lesions in both cerebral hemispheres as well as the absence of the flow void in both ICAs on T2 weighted axial images. Digital substraction angiography (DSA) showed complete occlusion of the bilateral internal carotid arteries just rostral to the bifurcation (fig l).

After consultation with the rheumatology clinic, a pathergy test was performed to

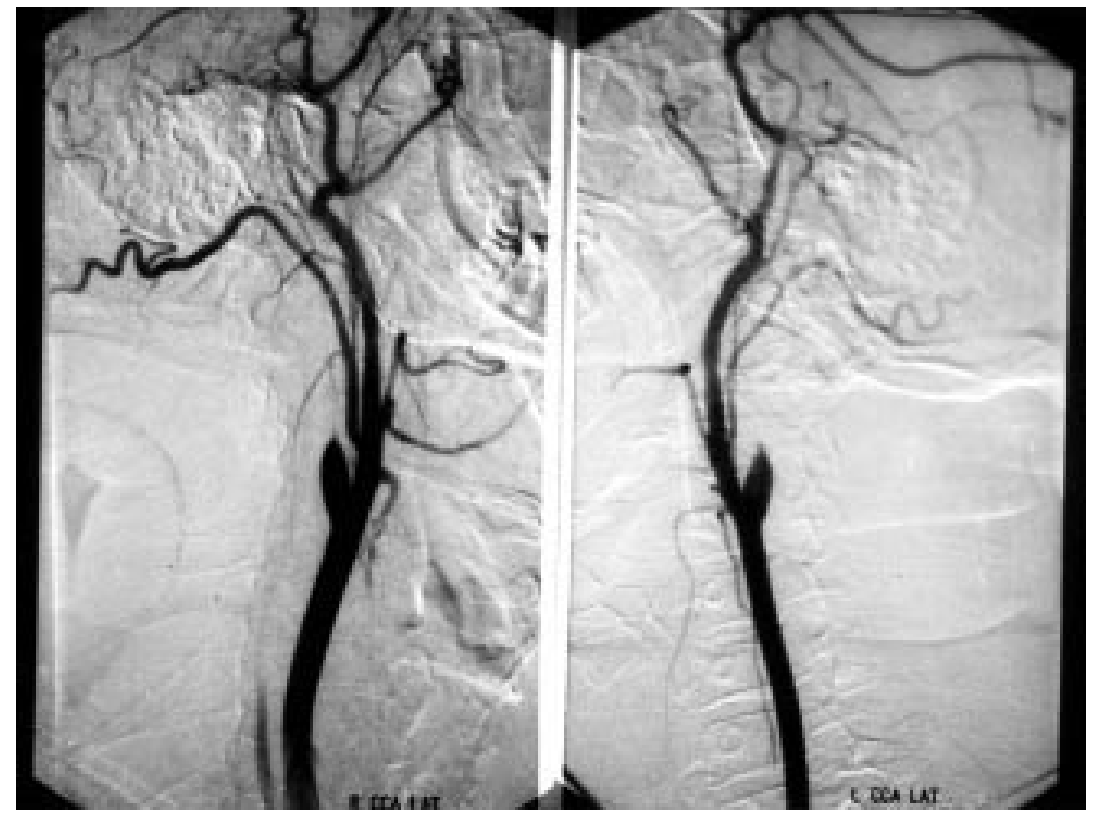

Figure 1 DSA of the cranial vessels showing the obstruction of $(A)$ right and $(B)$ left internal carotid arteries on lateral view.

confirm the diagnosis of $\mathrm{BD}$ and found to be positive. The patient was then transferred to the rheumatology clinic. He was treated with aspirin $300 \mathrm{mg} / \mathrm{day}$, prednisolon $1 \mathrm{mg} / \mathrm{kg} / \mathrm{day}$, pentoxifylline $1200 \mathrm{mg} /$ day, $750 \mathrm{mg}$ pulse cyclophosphamide monthly for BD. He was also treated with oral antibiotics and analgesics for the epididymitis. Two months later, he had almost completely recovered.

\section{Comment}

Our patient had presented with unusual neurological findings for a classic stroke syndrome and MRI showed bihemispheric ischaemic lesions and bilateral ICA occlusion, which was also shown by DSA. It is known that cardiovascular risk factors, smoking, fibromuscular dysplasia, or moyamoya disease are frequently found as an aetiological factor in patients with bilateral ICA occlusion, whereas essential thrombocytaemia, giant cell arteritis, and $\mathrm{BD}$ are among the very rare causes. ${ }^{2}$

Although our patient did not have cardiovascular risk factors except for smoking, he had been suffering from BD for about 10 years, which was not diagnosed before neurological presentation. His medical history, skin lesions, and urogenital findings supported with a positive pathergy test verified the diagnosis of $\mathrm{BD}$ according to latest diagnostic criteria for $\mathrm{BD}{ }^{4}$

Neurological involvement in $\mathrm{BD}$ has been reported to occur in $2.2 \%$ to $43 \%$ of cases in large series, either in the form of neuroBehçet disease (parenchymal CNS involvement) or vasculo-Behçet disease (secondary or non-parenchymal CNS involvement) or both. ${ }^{15}$ Neuro-Behçet's disease has a characteristic clinical picture with male predominance and typical cranial MRI findings of reversible inflammatory parenchymal lesions, attributable to small vessel disease, which may rarely be confused with those of MS. ${ }^{5}$ On the other hand, vasculo-Behçet's disease is attributable to large vessel disease generally in the form of cerebral venous thrombosis and has limited symptoms with a better prognosis. ${ }^{15}$ Our patient's neurological signs and symptoms were highly suggestive of
neuro-Behçet; however CSF findings with acellularity and normal protein level and neuro-imaging studies showing ischaemic lesions and bilateral ICA occlusions supported a very unusual type of vasculo-Behçet.

Diffuse cerebral atrophy and survival with minimal or no neurological deficit as in our patient is not infrequent in patients with bilateral ICA occlusion. This is explained by the adequate collateral flow provided by vertebrobasilar system and slow, gradual occlusion.

Occlusive lesions in the bilateral ICAs, as seen in our patient, are extremely rare in $\mathrm{BD}$ and we suggest that this is a very unusual case of vasculo-neuro-Behçet's disease. We also conclude that BD should always be remembered as an aetiological factor for bilateral ICA occlusions, especially in countries where the disease is highly prevalent.

A Sagduyu, H Sirin Neurology Department, Ege University, Turkey

F Oksel, T Turk

Rheumatology Department, Ege University

D Ozenc

Neurosurgery Department, Ege University

Correspondence to: $\operatorname{Dr}$ A Sagduyu, Ege University Medical School Hospital, Neurology Department, Bornova- IZMIR, Turkey; sagduyu@med.ege.edu.tr

\section{References}

1 Siva A. Vasculitis of the nervous system. Neurol 2001;248:451-68.

2 Catala M, Rancurel G, Raynaud C, et al. Bilateral occlusion of the internal carotid arteries. Analysis of a series of 19 patients. Rev Neurol 1995; 151:648-56.

3 Ohori N, Toda K, Ohta S, et al. A case of Behçet's disease presenting cerebral infarction due to occlusions of the bilateral internal carotid arteries. Rinsho Shinkeigaku 1999;39:856-9

4 International Study Group for Behçet's Disease. Criteria for diagnosis of Behcet's disease. Lancet 1990;335:1078-80.

5 Akman-Demir G, Serdaroglu P, Tasci B and the Neuro-Behçet Study Group. Clinical patterns of neurological involvement in Behchet's disease: evaluation of 200 patients. Brain 1999;122:2171-81. 


\section{Miller-Fisher syndrome and Hodgkin's disease}

Miller-Fisher syndrome (MFS) is a rare clinical entity classically regarded as a variant of Guillain-Barré syndrome (GBS) and characterised by the clinical triad of ophthalmoplegia, ataxia and areflexia. ${ }^{1}$ In MFS, paralysis is restricted to extraocular and occasionally other craniobulbar muscles. We report on a patient with a relapsing Hodgkin's disease who developed MFS. Conventional immunosuppressive and intravenous immunoglobulin treatments improved the neurological deficits.

This 27 year old white man who had an eight year history of Hodgkin's disease (type mixed cellularity, pathological stage IVB) had been receiving a salvage ESHAP regimen (etoposide VP-16 $68 \mathrm{mg} /$ day, methylprednisolone $500 \mathrm{mg} /$ day, and cisplatin $42.5 \mathrm{mg}$ / day for four days and cytosinearabinoside $3.4 \mathrm{~g} /$ day on the fifth day) since the first disease relapse four months before admission. He was admitted to the hospital for constitutional symptoms $\left(39^{\circ} \mathrm{C}\right.$ fever, recurrent nigh sweats, fatigue, malaise, and weakness). There was no history of infection. General examination was unremarkable except for bilateral inguinal adenopathy $(1.5 \times 1.5 \mathrm{~cm})$. Haemoglobin concentration was $63 \mathrm{~g} / \mathrm{l}$, packed cell volume $17.8 \%$, platelet count $89 \times 10^{9} / 1$, white cell count $3.34 \times 10^{9} / 1$ (neutrophils $2.42 \times 10^{9} / 1$ ), and lactate dehydrogenase $461 \mathrm{U} / 1$. Results of the following investigations were normal: glucose, cholesterol triglycerides, and ions; renal, liver, and thyroid function tests; vitamin B12 and folic acid; and tests for Campylobacter jejuni, herpes simplex virus, herpes zoster virus, cytomegalovirus, Epstein-Barr virus, Streptococcus pyogenes, Borrelia sp, syphilis, and cerebrospinal fluid parameters

Staging evaluation included negative computed tomography of the chest. Computed tomography of the abdomen showed paraaortic nodal enlargement and normal sized spleen. Bone marrow examination found histological evidence of Hodgkin's disease. Therefore, a diagnosis of relapsing Hodgkin's disease was considered.

Before starting a cycle of ESHAP chemotherapy, the patient complained of bilateral horizontal diplopia, photophobia, dysphonia and gait instability. Neurological function was assessed at that time, eight days after admis sion. Examination of the cranial nerves found a left sided ptosis with a total bilateral external ophthalmoplegia and fixed dilated pupils The patient's pupillary response to a $0.05 \%$ solution of pilocarpine showed increased sensitivity consistent with a postganglionic parasympathetic lesion. (Oculomotor nerves are among the few myelinated fibres of the postganglionic nervous system and this patient likely had dysfunction in these fibres similar to that observed in the other peripheral nerves. Pupillary abnormalities are encountered in about half of patients with MFS. ${ }^{2}$ ) There was dysphonia, mild dysphagia, and peripheral seventh nerve palsy. Examination of the peripheral nervous system showed loss of deep tendon reflexes. His muscle strength was normal, and pinprick, touch, position, and vibratory sensation were not impaired. There was obvious ataxia in all four limbs. He could walk with assistance and tandem gait was impossible. His cerebrospinal fluid protein concentration was $0.79 \mathrm{~g} / \mathrm{l}$ with 2 lymphocytes $/ \mathrm{mm}^{3}$. Cerebrospinal fluid culture and cytological studies showed only normal lymphocytes. Subsequent investigations found increased IgG ganglioside antibodies to
GQ1b glycolipids (titre of 4900). Standard delayed hypersensitive skin tests were performed to purified protein derivative of tuberculin (intermediate strength), Candida albicans, mumps, tricophyton, and streptokinase/ streptodornase, showing failure to elicit a response to any skin test antigens. Serum immunoglobulin concentrations were increased (IgG: $19 \mathrm{~g} / \mathrm{l}$, normal $10.51 \pm 2.9$, IgA: $4.8 \mathrm{~g} / \mathrm{l}$, normal $1.65 \pm 0.8$ ).

Gadolinium enhanced magnetic resonance imaging of the head showed no abnormalities. There was neurophysiological evidence of an axonal sensory neuropathy (sensory conduction in the right sural and median nerves was absent; the right median motor compound muscle action potential was $7.1 \mathrm{mV}$ with a conduction velocity of $41.5 \mathrm{~m} / \mathrm{s}$ ). F wave latencies from the right posterior tibial, right common peroneal, right median, and ulnar nerves were minimally prolonged two days after onset but were within normal limits by three months. The patient presented moderate reduction of facial compound muscle action potential amplitude (right amplitude: $1.5 \mathrm{mV}$, left amplitude: $1.3 \mathrm{mV}$, right latency: $3 \mathrm{~ms}$, left latency: $3.2 \mathrm{~ms}$ ). Blink reflex Rl latencies were mildly prolonged (right: $13.9 \mathrm{~ms}$, left: $14 \mathrm{~ms}$ ). Blink R2 response latencies were normal (right: $30 \mathrm{~ms}$, left: $29 \mathrm{~ms}$ ). Masseter reflex was normal. The amplitude of the distal sensory evoked response was greatly reduced (upper extremity somatosensory evoked potentials to median nerve stimulation at the wrist). Brainstem auditory evoked potentials were normal Intravenous immunoglobulin was given for five days at a dosage of $0.4 \mathrm{~g} / \mathrm{kg} /$ day, starting 24 hours after the onset of neurological symptoms. He gradually improved over the next two weeks. A follow up examination by the time of discharge four weeks after the onset found nearly complete clinical recovery from the ataxia and occasional diplopia but the tendon reflexes were still hypoactive. Three months later, neurological examination and lumbar puncture results were normal, all electrophysiological parameters were normalised, and IgG antibody titres to GQIb were not detectable.

In Hodgkin's disease, the incidence of polyneuropathy is about the same as for the reticuloses in general-that is, approximately 1 or $2 \% .^{3}$ The major clinical picture of this patient was acute ataxia, ophthalmoplegia, and areflexia associated with increased cerebrospinal fluid protein and high titres of antibodies to the GQlb ganglioside in the context of relapsing Hodgkin's disease, which suggests an autoimmune mediated neurological disorder. To our knowledge this is the first report on a patient with MFS evolving during a relapse of Hodgkin's disease. GBS and MFS occur in relation with conditions marked by partial immunosuppression, such as systemic lupus erythematosus, pregnancy, postoperative states, and a host of viral infections. ${ }^{3}$ Such situations are commonplace, and yet only a tiny proportion is complicated by GBS or MFS. This suggests that a special set of circumstances must prevail for MFS and GBS to occur. Viewed this way, not only are MFS and GBS disorders that can occur in the presence of partial immunosuppression, but also the immunosuppression may be involved in the pathogenesis of the syndromes. One must ask how an autoimmune, possibly cell mediated reaction can occur in an immunosuppressed patient. Animal models such as the NZB mouse show that depression of cell mediated immunity and the $\mathrm{T}$ cell system is associated with an increase in autoantibodies and autoimmune diseases, even though this increase is more often humorally mediated.$^{45}$. Lisak et $a l^{3}$ described three patients with GBS and Hodgkin's disease, postulating that selective depression of cell mediated immunity from whatever cause may allow the development of an immune reaction, either humoral, cellular, or both, directed against peripheral nervous system antigens.

The development of MFS in the context of relapsing Hodgkin's disease, together with the improvement of this syndrome after tumour treatment and intravenous immunoglobulins, supports the theory that partial immunosuppression and the presence of IgG anti-GQlb are possible pathogenic mechanisms.

E Rubio-Nazabal, J Marey-Lopez, J P Torres-Carrete, P Alvarez-Perez, P Rey Del Corral

Departments of Neurology and Haematology, Hospital Juan Canalejo, La Coruña, Spain

Competing interests: none declared

Correspondence to: Dr E Rubio-Nazabal, Servicio de Neurología, Hospital Juan Canalejo, As Xubias sn, 15006 La Coruña, Spain; jmareyl@telefonica.net

\section{References}

1 Fisher M. An unusual variant of acute idiopathic polyneuritis (syndrome of ophthalmoplegia, ataxia and areflexia). $N$ Engl J Med 1956;255:57-65.

2 Keane JR. Tonic pupils with acute ophthalmoplegic polineuritis. Ann Neurol 1977;2:393-6.

3 Lisak RP, Mitchell M, Zweiman B, et al. Guillain-Barré syndrome and Hodgkin's disease: three cases with immunological studies. Ann Neurol 1977:1:72-8.

4 Leventhal BG, Talal N. Response of NZB and NZB-NZW spleen cells to mitogenic antigens. J Immunol 1970; 104:918-23.

5 Churchill WH, Rocklin RE, Maloney WC. In vitro evidence of normal lymphocyte function in some patients with Hodgkin's disease and negative delayed cutaneous hypersensitivity. Natl Cancer Inst Monogr 1973;36:99-106.

\section{Neuromyotonia and myasthenia gravis without thymoma}

Neuromyotonia is a syndrome characterised by motor unit hyperactivity leading to muscle cramps, fasciculations, muscle stiffness, and persistent muscle contraction. In most neuromyotonia patients, the disorder is acquired An autoimmune or paraneoplastic origin is common. ${ }^{1-3}$ Myasthenia gravis, thyrotoxicosis, systemic sclerosis, inflammatory demyelinating neuropathies, thymoma, bronchial carcinoma, and small cell lung cancer may be associated. Here, we report a patient with neuromyotonia, associated with myasthenia gravis and anti-voltage-gated potassium channels (VGKC) and anti-acetylcholine receptor (AChR) antibodies without thymoma.

A 58 year old man of Portuguese descent presented at our neuromuscular clinic with dysesthesia and hypesthesia in the first three fingers of the right hand. Symptoms had started nine years before and had been attributed to cervical radiculopathy. Over the years, the symptoms had been fluctuating but for the past two months they had become debilitating. Therefore, the patient sought a second opinion. The patient volunteered that, although right hand pain was his main complaint, for many years his hands and feet were swollen and red. There was stiffness and loss of dexterity of all fingers. He had difficulty writing, using scissors, and using a handheld 


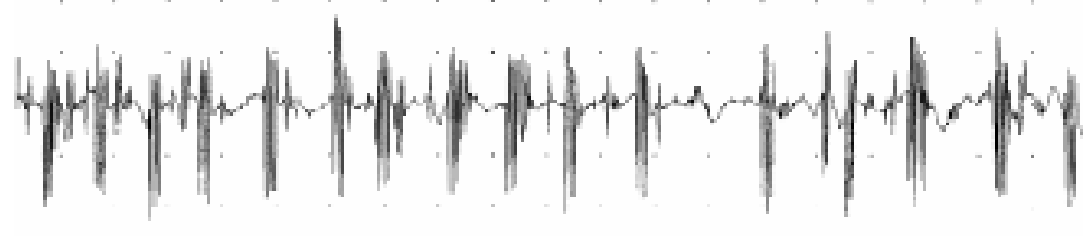

$200 \mu \mathrm{V}$

$50 \mathrm{~ms}$

Figure 1 Myokymic discharges recorded at rest with a concentric needle electrode from the right dorsal interosseus muscle, shown at two different sweep speeds.

computer. Frequent cramps occurred in the fingers and toes. There was painful tension in the calfs, the feet, and the hands. The patient also complained of excessive sweating. These symptoms had progressively worsened. One year before presenting to us, he developed ptosis of the right upper eyelid, rapidly followed by vertical and horizontal diplopia. These symptoms were fluctuating with worsening in the evening. Repetitive stimulation of the facial nerve showed a decremental response, symptoms and signs disappeared after injection of prostigmine, and anti-AChR antibodies were found. It was concluded that the patient had ocular myasthenia and the patient was treated with oral methylprednisolone. Improvement was rapid and after a few weeks treatment was stopped. Two weeks before presentation, the patient again complained of right palpebral ptosis and diplopia. The symptoms were responsive to pyridostigmine bromide. The medical history was remarkable for ophthalmical migraine, arterial hypertension, and hypercholesterolaemia. Treatment consisted of fenofibrate and metoprolol. The family history was non-contributory.

On clinical examination, continuous undulating movements were noted in the small muscles of hands and feet and in the orbicularis oculi muscles. Small amplitude, involuntary movements of fingers and toes were conspicuous at rest. The fingers were stiff and the patient had difficulty performing rapid alternating movements with his fingers. Tactile and pain sensation was diminished only in the firs three fingers of the right hand. Tinel's and Phalen's signs were present at the right wrist and there was right hand grip weakness. Right upper eyelid ptosis, rapidly increasing on upward gaze was noted. Horizontal diplopia occurred in right lateral and vertical gaze directions. Deep tendon reflexes were normal and plantar responses were flexor.

Complete blood count, serum creatinine, blood urea nitrogen, liver function tests, serum electrolytes, thyroid function tests, and serum creatine kinase were normal. Rheumatoid factor was negative and there were no antibodies against striated muscle, but antinuclear antibodies were positive at a titre of $1 / 80$. Prostate specific and carcinoembryonic antigens were negative. Both AChR antibodies (26 nmol $/ \mathrm{ml}$, normal values less than 0.5 $\mathrm{nmol} / \mathrm{ml}$ ) and VGKC antibodies (1091 pmol/l (normal values less than $100 \mathrm{pmol} / \mathrm{l}$ ) were detected. Computed tomography of the chest was normal.

Nerve conduction studies showed evidence of a severe rightsided carpal tunnel syndrome, but otherwise they were normal. Needle electromyography revealed myokymic discharges in distal muscles of upper and lower extremities (fig 1). These discharges consisted of bursts of motor unit potentials, appearing as doublets, triplets, or multiplets with intraburst frequencies of 40 to $100 \mathrm{~Hz}$. Burst recurrence was irregular with an interburst frequency of $5-8 \mathrm{~Hz}$. There was evidence of mild chronic denervation with slightly reduced recruitment in distal muscles.

Anti-VGKC antibodies are found in approximately $40 \%$ of patients with acquired neuromyotonia ${ }^{-4}$; they are also found in patients with other neuromuscular hyperexcitability syndromes, such as cramp fasciculation syndrome, acquired rippling muscle syndrome, facial myokymia. ${ }^{56}$ In a significant proportion of these patients, coexistence of myasthenia gravis and neoplastic disorders, thymoma in particular, is observed. ${ }^{45}$ About $20 \%$ of all reported neuromyotonia patients had thymoma; $70 \%$ thereof also had myasthenia gravis and anti-AChR antibodies and 20\% had anti-AChR antibodies without overt myasthenia gravis. The absence of antistriated muscle antibodies and of radiological evidence of mediastinal tumour in a patient with neuromyotonia of nine years duration illustrates that the association of autoimmune neuromyotonia and myasthenia gravis can occur without thymoma.
A Vincent

Neurosciences Group, Department of Clinical Neurology, Institute of Molecular Medicine, University of Oxford, John Radcliffe Hospital, Oxford, UK

Correspondence to: Dr P Van den Bergh, Service de Neurologie, Cliniques universitaires Saint-Luc, Université catholique de Louvain, avenue Hippocrate 10, 1200 Brussels, Belgium; vandenbergh@nchm.ucl.ac.be

Conflicts of interest: The University of Oxford on behalf of $A V$ receives consulting fees from RSR Ltd who market AchR antibody assays

\section{References}

1 Newsom-Davis J, Mills R. Immunological associations of acquired neuromyotonia. Brain 1993;116:453-69.

2 Vincent A. Understanding neuromyotonia. Muscle Nerve 2000;23:655-7.

3 Gutmann Lu, Libell D, Gutman La. When is myokymia neuromyotonia? Muscle Nerve 2001;24:151-3.

4 Hart I, Vincent A, Willison H. In: Engel AG, ed. Myasthenia gravis and myasthenic disorders. New York: Oxford University Press, 1999:229-50

5 Vernino S, Lennon VA. Neuronal ion channel antibodies in patients with neuromuscular hyperexcitability. Neurology 2001;56:A231.

6 Gutmann L, Tellers JG, Vernino S. Persistent facial myokimia associated with $\mathrm{K}+$ channel antibodies. Neurology 200 1;57:1707-8.

\section{Acute attacks and brain stem} signs in a patient with glutamic acid decarboxylase

\section{autoantibodies}

Glutamic acid decarboxylase (GAD) is a major autoantigen in type I diabetes mellitus and stiff-man syndrome. Patients with progressive cerebellar ataxia and GAD autoantibodies (GAD-Abs) have been reported, ${ }^{12}$ and the pathogenetic role for GAD-Abs in suppressing cerebellar $\gamma$-aminobutyric-acid (GABA)-ergic transmission has been discussed. We present a woman who eventually developed progressive cerebellar ataxia, but had stroke-like episodes and brain stem involvement during her clinical course.

A 63 year old woman suffered dizziness of sudden onset accompanied by nausea and vomiting. Her physician found horizontal, gaze evoked nystagmus. A few days later, she noticed transient horizontal diplopia, after which spontaneously all her symptoms gradually subsided. Two months later, she experienced intermittent vertigo when she turned her head and then unsteadiness of gait. Her past medical and family histories were unremarkable. On examination, she was fully conscious and had no general physical abnormalities. There was coarse horizontal nystagmus, coarser on the left side. On phonation, her posterior pharyngeal wall shifted rightward, indicating paralysis of the constrictor muscles of the left side of the posterior pharyngeal wall (signe de rideau, Vernet $\left.^{3}\right)$. She had ataxia in her left arm and leg and walked throwing the left leg outward. Although lesion in the left dorsolateral lower brain stem was suspected, MRI and MR arterial and venous images were unremarkable. A routine blood examination, as well as glucose tolerance and thyroid function tests, detected no abnormalities. CSF analysis was normal with negative oligoclonal IgG bands and a 
normal IgG index of 0.45 . Her condition remained unchanged for six months, after which gait unsteadiness progressed gradually for one month. Thereafter, she had difficulty in speaking and swallowing on waking in the morning. In addition to the signs seen at the first presentation, a neurological examination showed ataxic dysarthria and limb ataxia on both sides. She became dependent on walking aids. The muscular tone of her limbs was decreased but the strength was normal. Tendon reflexes were normal, and planta responses flexor on both sides. There was neither sensory nor bladder disturbance. Repeat CSF analysis and brain MRI results were normal. An electroencephalogram and nerve conduction studies gave normal results.

Routine haematological and blood chemistry studies, as well as the serum levels of vitamins B1, B12, and E, were normal. Faecal occult blood was negative. Infection by neurotrophic viruses was excluded serologically. Polymerase chain reaction analysis of the CSF for herpes simplex virus types 1 and 2 was negative. Intensive search for gynaecological, breast, or lung cancer, as well as haematological malignancies, including whole body com puted tomography, bilateral mammography, and bone and gallium scintigrams produced negative results; anti-Hu and Yo antibodies were negative. Genetic analysis for spinocerebellar ataxia type 6 was negative. Glucose tolerance was impaired, but insulin secretion preserved. The serum GAD-Abs level determined by radioimmunoassay was highly increased at $10400 \mathrm{U} / \mathrm{ml}$ (normal < $1.5 \mathrm{U} / \mathrm{ml}$ ). Evaluation of GAD-Abs from plasma frozen at her first presentation showed a titre of 9830 $\mathrm{U} / \mathrm{ml}$. Serum thyroid stimulating hormone was slightly increased, but thyroid hormone levels were normal, indicative of subclinica hypothyroidism associated with autoantibodies to thyroperoxydase and thyroglobulin. Low titre positivities were found for antinuclear, anti-double stranded DNA, anti-parietal cells, and anti-insulin antibodies. CSF GAD-Abs titre was $496 \mathrm{U} / \mathrm{ml}$. Intrathecal GAD-Ab synthesis, calculated by Schüller's formula, gave a ratio of 10.7 for intrathecal GAD-Ab specific activity (ASA)/serum ASA, consistent with positive intrathecal synthesis.

Her limb and gait ataxia progressed and were overlaid by truncal ataxia within a month. She underwent a five time course of double filtration plasmapheresis that filtered 15 litres of plasma. Immediately after completion of the plasmapheresis course, her GADAbs titre decreased to $4700 \mathrm{U} / \mathrm{ml}$, and left posterior pharyngeal wall motion and independent gait returned. Ataxia, however returned three weeks later and then progressed, accompanied by a gradual rise in GAD-Abs titre. A five day course of intra venous immunoglobulins $0.4 \mathrm{~g} / \mathrm{kg} /$ day produced no improvement.

The overall clinical picture for this patient subacutely progressive ataxia, is complicated by acute onset, exacerbations, and such signs of brain stem involvement as hemiparalysis of the posterior pharyngeal wall and asymmetrical coarse nystagmus. Although she does not have type I diabetes mellitus, the high serum GAD-Abs titre, intrathecal GAD-Ab synthesis, and presence of organ specific autoantibodies are comparable to previous findings for patients with progressive cerebellar ataxia and GAD-Abs. ${ }^{12}$ Selective suppression of GABA-ergic transmission by GAD-Abs is possible cause of SMS, cerebellar ataxia, focal epilepsy, and palatal myoclonus. This mech anism, however, does not explain our patient's paralysis of the pharyngeal constrictor mus- cles because motoneurons in the nucleus ambiguus receive GABA mediated inhibition. ${ }^{4}$ As speculated by Honnorat et al, high GADAbs titre would merely reflect the presence of a more complex immune reaction against the nervous system. In this context, the subacute and atypical presentation of this patient raises the possibility that the GAD-Abs might have been a paraneoplastic phenomenon. Sillevis Smitt et al reported reversible cerebellar ataxia attributable to autoantibodies against a glutamate receptor in two patients with Hodgkin's disease. ${ }^{5}$ At present, however, follow up examinations of this patient showed no evidence for malignancy. The case of our patient suggests that progressive ataxia with high GAD-Abs titre may present with episodes that resemble multiple sclerosis or recurrent brain stem encephalitis.

S Matsumoto, T Kusuhara, M Nakajima, S Ouma, M Takahashi, T Yamada Department of Neurology, School of Medicine, Fukuoka University, Japan

Correspondence to: Dr M Nakajima, Department of Neurology, School of Medicine, Fukuoka University, 7-45-1 Nanakuma, Johnan-ku, Fukuoka, Japan 814-0180 masashi@fukuoka-u.ac.jp

\section{References}

1 Saiz A, Arpa J, Sagasta A, et al. Autoantibodies to glutamic acid decarboxylase in three patients with cerebellar ataxia, late-onset insulin-dependent diabetes mellitus, and polyendocrine autoimmunity. Neurology 1997;49:1026-30.

2 Honnorat J, Saiz A, Giometto B, et al. Cerebellar ataxia with anti-glutamic acid decarboxylase antibodies. Arch Neurol 2001;58:225-30

3 Vernet $M$. Les paralysies laryngés associées. Thèse. Lyon: 1916:49-55.

4 Yajima Y, Hayashi Y. GABA(A receptor-mediated inhibition in the nucleus ambiguus motoneuron. Neuroscience 1997;79: 1079-88.

5 Sillevis Smitt $\mathbf{P}$, Kinoshita A, De Leeuw B, et al. Paraneoplastic cerebellar ataxia due to autoantibodies against a glutamate receptor. N Engl J Med 2000;342:21-7.

High concentrations of sVCAM- 1 and SICAM-1 in the cerebrospinal fluid of patients with intracerebral haemorrhage are associated with poor outcome

Intracerebral haemorrhage (ICH) accounts for approximately $10 \%$ of strokes and is a life threatening condition with a 30 day mortality rate of about $45 \% .{ }^{1}$ The adhesion molecules intercellular adhesion molecule-1 (ICAM-1) and vascular cell adhesion molecule-1 (VCAM-1) are proinflammatory parameter for the activation of the immune system. ${ }^{2}$ They have been correlated with acute inflammation in several systemic and neurological inflammatory diseases. Recently, it was suggested that an inflammatory reaction is responsible for reperfusion damage leading to brain damage and tissue destruction after acute ischaemia and subarachnoid haemorrhage. ${ }^{34}$ In this study, we investigated whether ventricular cerebrospinal fluid (CSF) and serum concentrations of adhesion molecules can be used as prognostic markers for the clinical outcome of patients with ICH.

For this purpose, we studied prospectively 10 patients with acute ICH and ventricular tamponade. Estimated blood volume of the ICH was between 40 and $60 \mathrm{ml}$ in all patients. Initial intubation and mechanical ventilation due to coma were required in all patients. All of them were being treated at the neurological intensive care unit after neurosurgical application of a ventricular drainage to treat acute hydrocephalus. Paired serum and CSF samples from the ventricular drainage were obtained within eight hours after the first symptoms attributed to ICH and within three hours after operation. Concentrations of soluble ICAM-1 (sICAM-1) and SVCAM-1 were determined by enzyme linked immunosorbent assay (ELISA). In corresponding clinical examinations, the Scandinavian stroke scale and Glasgow coma scale scores were determined. The patients were categorised into two groups: patients who survived $(n=6)$ and patients who died $(n=4)$ from cerebral causes within eight weeks after the onset of ICH. Patients with prior cerebrovascular diseases and patients who subsequently died of non-cerebral causes were excluded from this pilot study. Data were analysed using the SPSS statistical program (SPSS, Chicago, Illinois, USA). The Wilcoxon test was applied to compare the two patient groups.

The two patient groups (surviving versus non-surviving) did not differ statistically with regard to age, sex, location and size of $\mathrm{ICH}$, and initial Glasgow coma scale and Scandinavian stroke scale scores. As fig 1 shows, the CSF concentrations of sICAM-1 were below $13.7 \mathrm{ng} / \mathrm{ml}$ (mean (SD) $8.7(4.7) \mathrm{ng} / \mathrm{ml}$ ) and of sVCAM-1 below $35.4 \mathrm{ng} / \mathrm{ml}$ ( 11.5 (13.1) ng/ $\mathrm{ml}$ ) in the group of patients who survived $(\mathrm{n}=6)$. However, in patients with a lethal outcome $(n=4)$, initial ventricular CSF concentrations of sICAM-1 were above $18.3 \mathrm{ng} /$ $\mathrm{ml}(25.5(9.3) \mathrm{ng} / \mathrm{ml})$ and of sVCAM-l were above $44.5 \mathrm{ng} / \mathrm{ml}$ (76.8 (45.0) ng/ml). These differences were significant for the CSF concentrations of sICAM-1 $(\mathrm{p}<0.01)$ and of
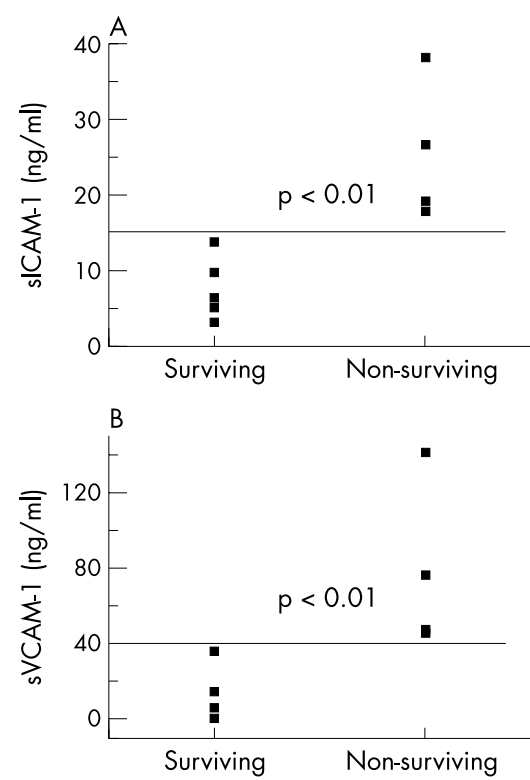

Figure 1 Ventricular cerebrospinal fluid concentrations of $(A)$ soluble intercellular adhesion molecule-1 (sICAM-1) and (B) soluble vascular cell adhesion molecule-1 (sVCAM-1) in 10 patients who had intracerebral haemorrhage with ventricular tamponade. The patients are categorised into two groups: patients who survived $(n=6)$ and patients who died $(n=4)$ from cerebral causes within eight weeks after the onset of intracerebral haemorrhage. 

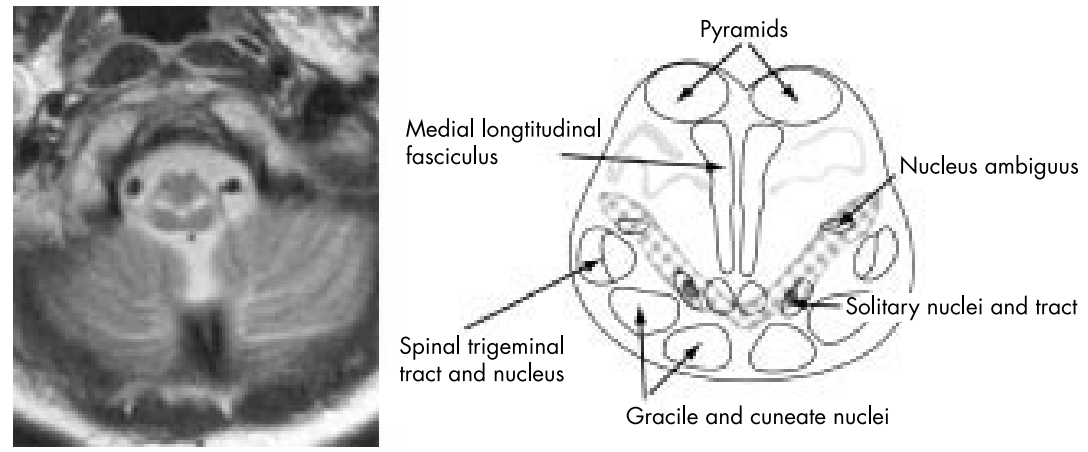

Figure 1 Left: T2 weighted axial MRI through the medulla. Right: diagram showing relevant medullary components.

sVCAM-1 $(\mathrm{p}<0.01)$. However, the concentrations of adhesion molecules in serum did not differ significantly (non-surviving: 444 (152) $\mathrm{ng} / \mathrm{ml}$ for sICAM-l, $1422(465) \mathrm{ng} / \mathrm{ml}$ for sVCAM-1; surviving: 463 (110) ng/ml for sICAM-1, 1147 (382) ng/ ml for SVCAM-1).

This is the first study to investigate soluble adhesion molecules in CSF and serum in patients with ICH with ventricular tamponade. We found a strong correlation between clinical outcome and the concentrations of soluble adhesion molecules in the CSF of patients with acute ICH and ventricular drainage. Moreover, we found more than threefold increases of SICAM-1 and of SVCAM-1 in the CSF of patients with lethal outcome as compared with CSF concentrations from patients with multiple sclerosis (s-ICAM-1: $2.8 \mathrm{ng} / \mathrm{ml}$, range $0.9-12.7$ sVCAM-1: $4.2 \mathrm{ng} / \mathrm{ml}$, range 0-21.5) and from healthy donors ( $\operatorname{sICAM}-1: 5.2(2.2) \mathrm{ng} / \mathrm{ml}$ ) as determined in our laboratory by identical test systems. ${ }^{25}$ The finding that the soluble adhesion molecules were increased in CSF but not in serum may indicate that the process leading to poor outcome occurs predominately in the brain. There are two possible explanations for the origin of increased CSF concentrations of soluble adhesion molecules. Firstly, brain tissue destruction may lead primarily to the release of adhesion molecules due to necrotic destruction. Secondly, ICH may initiate an inflammatory process leading to secondary brain damage, as has been suggested in human ischaemic stroke, ${ }^{4}$ as well as for experimental ICH and subarachnoid haemorrhage in animal models. ${ }^{3}{ }^{3}$ With regard to the second hypothesis, it would be interesting to investigate the effects of early antiinflammatory treatment in patients with ICH and an initial highly increased concentration of adhesion molecules in their ventricular CSF samples. In this condition, early application of corticosteroids may be useful to suppress the deviating inflammatory reaction. ${ }^{2}$ The blockage of ICAM-1 and VCAM-1 by systemic treatment with monoclonal antibodies would probably not be helpful, as the pathogenetic concept is to block the migration of inflammatory cells into the central nervous system. However, based on our results, it can be speculated that these cells are already inside the central nervous system and thus out of reach of these antibodies.

With these data of only 10 patients, it canno finally be concluded whether the increased soluble adhesion molecules in CSF are indicators of the fatal process or are responsible for the initiation of secondary brain damage.

\section{Acknowledgements}

Dr B Engelhardt is gratefully acknowledged for critically discussing the manuscript.
*J Kraus, P Oschmann

Department of Neurology, Justus-Liebig University Giessen, Am Steg 14, 35385 Giessen, Germany

S Leis, B Neundörfer, J G Heckmann

Department of Neurology, Friedrich-Alexander University Erlangen-Nuremberg, Schwabachanlage 6, 91054 Erlangen, Germany

*Also the Department of Vascular Cell Biology, Max Planck/WG Kerckhoff-Institute Bad Nauheim, Parkstrasse 1, 61231 Bad Nauheim, Germany

Correspondence to: Dr J Kraus; joerg.r.kraus@neuro.med.uni-giessen.de

\section{References}

1 Gong C, Hoff JT, Keep RF. Acute inflammatory reaction following experimental intracerebral hemorrhage in rat. Brain Res 2000;871:57-65.

2 Kraus J, Oschmann P, Engelhardt B, et al. Soluble and cell surface ICAM-1 as markers for disease activity in multiple sclerosis. Acta Neurol Scand 1998;98:102-9.

3 Bavbek M, Polin R, Kwan AL, et al. Monoclonal antibodies against ICAM-1 and CD1 8 attenuate cerebral vasospasm after experimental subarachnoid hemorrhage in rabbits. Stroke 1998;29:1930-5.

4 Lindsberg PJ, Carpen O, Paetau A, et al. Endothelial ICAM-1 expression associated with inflammatory cell response in human ischemic stroke. Circulation 1996;94:939-45

5 Kraus J, Engelhardt B, Chatzimanolis N, et al. Cell surface bound and soluble adhesion molecules in CSF and blood in multiple sclerosis: correlation with MRI-measures of subclinical disease severity and activity. $J$ Neuroimmunol 2002;122:175-85.

\section{Ondine's curse in a woman with Leber's hereditary optic neuropathy}

Leber's hereditary optic neuropathy (LHON) is a maternally inherited disease of mitochondrial DNA. Several mutation sites have been described. All have been associated with visual loss, but mutations at nucleotide position 11778,3460 , and recently 14484 , have also been associated with a multiple sclerosis (MS)-like disease. ${ }^{1-3}$

We report a woman with undiagnosed LHON who presented with life threatening ventilatory failure.

A 39 year old woman who had had bilateral synchronous severe visual loss to perception of light some two years earlier (see below), was admitted after a two week illness with a purulent cough. She was confined to bed and had received oral antibiotics from her general practitioner. She had a history of chronic headaches but reported no change in their frequency before presentation. On admission she was obtunded with a Glasgow Coma Scale (GCS) score of 3/15. She was hypoventilating, with a severe respiratory acidosis. Arterial blood gas (ABG) showed $\mathrm{pH}$ 7.04, $\mathrm{Po}_{2} 40.9$ $\mathrm{kPa}, \mathrm{PCO}_{2} 16.2 \mathrm{kPa}$, and bicarbonate $22 \mathrm{mmol} / \mathrm{l}$. She was admitted to an intensive care unit and ventilated with later tracheostomy. She was weaned from the ventilator after 31 days and transferred to a ward. Five days later she had a second respiratory arrest requiring further ventilation. She was transferred to another unit 73 days after admission for consideration of long term non-invasive ventilation.

This patient had consumed alcohol to excess and had been admitted previously for benzodiazepine overdose and complications of alcoholic liver disease. Two years earlier she had presented to an ophthalmologist complaining of two months of painless visual loss. Visual acuity was counting fingers bilaterally with central scotomata and absent pupil reactions. Fundoscopy showed bilateral disc oedema, dilated capillaries around the disc margins, and venous pulsations. A CT brain scan was normal, but the patient declined further investigation and a diagnosis of possible toxic amblyopia was made. There was no family history of visual loss. She had three siblings in their 30s, and three children aged 9-12 years who were well.

On examination after transfer (two months after her first respiratory arrest), she was alert, oriented, and breathing room air spontaneously. She was unable to stand and had globally wasted limbs consistent with prolonged illness. She could just perceive light bilaterally and both optic discs looked pale and the pupils were mid-dilated and unreactive. She had a divergent gaze in the primary position with coarse gaze evoked nystagmus in all directions. A jaw jerk was present and she had a mild facial diplegia with intact sensation. She could speak and swallow adequately and was able to cough and hold her breath to command. She had a spastic quadriparesis with grade $4 / 5$ power in the arms but weaker legs and a flicker of movement only at the toes. Anterior abdominal motion during breathing while lying supine was normal. Reflexes were brisk throughout and plantar responses were extensor. There was a subjective sensory abnormality to light touch to the mid-thighs and joint position sense was severely impaired in the feet but intact in the fingers. Breath sounds were quiet and chest excursion limited. She had a distended abdomen with a four finger breadth liver edge palpable and shifting dullness consistent with ascites. ABG on air showed pH 7.31, $\mathrm{Po}_{2} 6.8$ $\mathrm{kPa}, \mathrm{PCO}_{2} 10.5 \mathrm{kPa}$, and bicarbonate 34.8 $\mathrm{mmol} / \mathrm{l}$. Four hours later she became drowsy with a GCS of 8/15. Further ABG revealed pH 7.19, $\mathrm{Pao}_{2} 5.5 \mathrm{kPa}, \mathrm{PCO}_{2} 12.8 \mathrm{kPa}$, and bicarbonate $28.3 \mathrm{mmol} / \mathrm{l}$. After four hours of noninvasive intermittent positive pressure ventilation (NIPPV); $A B G$ on two litres of entrained oxygen showed $\mathrm{pH} 7.44, \mathrm{Po}_{2} 16.4$ $\mathrm{kPa}, \mathrm{PCO}_{2} 5.2 \mathrm{kPa}, \mathrm{HCO}_{3} 27.4 \mathrm{mmol} / \mathrm{l}$. She was subsequently transferred to a ward and treated with NIPPV, on room air, at a pressure of $14 \mathrm{~cm} \mathrm{H} \mathrm{H}_{2} \mathrm{O}$ overnight and during daytime naps.

An MRI scan of her brain showed symmetrical high signal lesions in the brainstem in the floor of the fourth ventricle at the level of the obex and in the medulla and upper cervical cord (fig 1). The remainder of the brain was spared and in particular there were no lesions suggestive of central pontine myelinolysis or alcoholic damage. CSF examination was unremarkable except for a marginally increased protein at $0.48 \mathrm{~g} / \mathrm{l}$. CSF and 
Table 1 Frequency of relapse of Guillain-Barré syndrome (GBS) and chronic inflammatory demyelinating polyradiculoneuropathy (CIDP) following various immunisations

\begin{tabular}{|c|c|c|c|c|}
\hline \multirow[b]{2}{*}{ Vaccine } & \multicolumn{2}{|l|}{ GBS } & \multicolumn{2}{|l|}{ CIDP } \\
\hline & Patients & Relapses & Patients & Relapses \\
\hline Influenza & 211 & $8(3.8 \%)$ & 46 & $2(4.3 \%)$ \\
\hline Tetanus & 105 & $6(5.7 \%)$ & 23 & $2(8.7 \%)$ \\
\hline Typhoid & 50 & $3(6.0 \%)$ & 14 & 0 \\
\hline Polio & 42 & $4(9.5 \%)$ & 7 & 0 \\
\hline Hepatitis A & 37 & $3(8.1 \%)$ & 7 & 0 \\
\hline Hepatitis B & 20 & $1(5.0 \%)$ & 2 & 0 \\
\hline Rabies & 1 & 0 & 0 & 0 \\
\hline Pneumococcus & 15 & 0 & 6 & $2(33.3 \%)$ \\
\hline BCG & 8 & $2(25.0 \%)$ & 4 & 0 \\
\hline Yellow fever & 12 & $2(16.7 \%)$ & 2 & 0 \\
\hline Meningococcus & 16 & $1(6.2 \%)$ & 4 & 0 \\
\hline Cholera & 5 & 0 & 0 & 0 \\
\hline Rubella & 5 & 0 & 1 & 0 \\
\hline Diphtheria & 5 & $2(40.0 \%)$ & 1 & 0 \\
\hline Measles & 2 & 0 & 0 & 0 \\
\hline Smallpox & 2 & 0 & 0 & 0 \\
\hline Mumps & 1 & 0 & 0 & 0 \\
\hline
\end{tabular}

Some patients had received more than one vaccine.

plasma lactate were both $2.1 \mathrm{mmol} / \mathrm{l}$ and oligoclonal bands were not found. DNA was extracted from a blood sample and analysed for mtDNA mutations using standard procedures and was negative at positions 3243 , 8344, 8993, 3460, and 14484, but with a homoplasmic mutation at position 11778 .

Our patient had the mutation most often associated with MS-like CNS lesions and visual loss in women. ${ }^{1}$ Brain stem lesions have been previously described in a patient with visual loss, complete ophthalmoplegia, and bilateral tinnitus. ${ }^{3}$ However, to our knowledge, this is the first description of LHON in association with brain stem lesions presenting with respiratory arrest and loss of involuntary ventilation (Ondine's curse). The high signal lesions in the pons and medulla involved the nucleus ambiguus and nucleus of the solitary tract, which are part of the ventral and dorsal respiratory groups respectively, and would seem well placed to account for loss of respiratory control during sleep with well preserved capacity for volitional respiratory manoeuvres while awake. Ondine's curse produced by lesions of these structures and their tracts through a variety of causes has been well described. ${ }^{45}$ However, the exact nature of CNS lesions in patients with mitochondrial cytopathy remains obscure.

Our patient tolerated NIPPV. She improved on this regimen such that 123 days after admission she was able to take a 45 minute daytime nap and maintain an oxygen saturation of $>97 \%$ throughout, while breathing room air unassisted. Eight months after her respiratory arrest, she was able to take a few steps with a Zimmer frame and had successfully weaned off NIPPV support. This patient provides a further example of the broad manifestations of mitochondrial disease.

\section{Acknowledgement}

We thank Dr M Hebden for permission to report a patient under his care.

M Sadler, C M Wiles

Department of Neurology, University Hospital of Wales, Cardiff, UK

N Stoodley

Department of Radiology, University Hospital of Wales
S J Linnane, A P Smith

Department of Respiratory Medicine, Llandough Hospital, Llandough, UK

Correspondence to: Dr M Sadler, Department of Neurology, University Hospital of Wales, Heath Park, Cardiff CF4 4XN, UK msadler@spirochete.net

\section{References}

1 Harding AE, Sweeney MG, Miller DH, et al. Occurrence of a multiple sclerosis like illness in women who have a Leber's hereditary optic neuropathy mitochondrial DNA mutation. Brain 1992; 1 15:979-89.

2 Riordan-Eva P, Sanders MD, Govan GG, et al. The clinical features of Leber's hereditary optic neuropathy defined by the presence of a pathogenic mitochondrial DNA mutation. Brain 1995; 118:319-37.

3 Funalot B, Ranoux D, Mas J-L, et al. Brainstem involvement in Leber's hereditary optic neuropathy: association with the 14484 mitochondrial DNA mutation. J Neurol Neurosurg Psychiatry 1996;61:533-4.

4 Devereaux MW, Keane JR, Davis RL. Automatic respiratory failure associated with infarction in the medulla. Arch Neurol 1973;29:46-52.

5 Tranmer BI, Tucker WS, Bilbao JM, et al. Sleep apnea following percutaneous cervical cordotomy. Can J Neurol Sci

1987;14:262-7.

\section{Risk of relapse of Guillain-Barré syndrome or chronic inflammatory demyelinating polyradiculoneuropathy following immunisation}

Reports of the rare occurrence of GuillainBarré syndrome (GBS) or chronic inflammatory demyelinating polyradiculoneuropathy (CIDP) following immunisation and recurrence of symptoms following subsequent immunisation $^{2}$ have given rise to concern over the safety of vaccine administration in this patient group. Similar concerns have been addressed and dismissed in patients with multiple sclerosis, ${ }^{3}$ but no such information exists for inflammatory neuropathy. To provide more information about vaccine safety in GBS and CIDP we audited the recurrence of neurological symptoms following immunisation.
The Guillain-Barré Syndrome Support Group, a British patient organisation, posted 3000 questionnaires to its members, asking them to identify their illness, record all immunisations administered after their illness, and describe any symptoms within six weeks of immunisation suggestive of recurrence of GBS or worsening of CIDP.

All but one of the patients who reported neurological symptoms after immunisation were contacted by telephone to confirm their history and to grade their symptoms using the modified Rankin scale. ${ }^{4}$ For the patient who could not be contacted by telephone, the patient's consultant neurologist provided the information. Questionnaires were sent to the general practitioner for each patient who reported a "relapse" to confirm which vaccine had been administered.

A total of 1114 patients $(37.1 \%)$ completed the questionnaires, of whom 927 had had GBS, 179 had CIDP, and eight were excluded because they had other diseases. Of the 927 patients with GBS, 311 had received immunisations since having GBS. Eleven (3.5\%, 95\% confidence limits (CL) $1.8 \%, 6.2 \%$ ) reported symptoms including increased fatigue, weakness, numbness, and paraesthesiae, but these were usually mild and no patient required hospitalisation or treatment. In three cases symptoms came on within 24 hours of immunisation and all but one developed symptoms within one week of immunisation. One patient reported symptoms rendering him unable to walk unaided or drive for six weeks, which increased his modified Rankin scale score from grade 2 to 4 .

Influenza, tetanus, and typhoid were the most common immunisations associated with a relapse after GBS but the number of patients who reported symptoms was small compared with the total numbers receiving each of these vaccines (table 1). Although the results suggest that some vaccines that are administered less frequently (such as diphtheria) may be associated with a higher relapse risk, the numbers were small and most of these vaccines were administered at the same time as other vaccines.

Of the 311 patients with GBS who had received vaccines after having GBS, 29 had also received a vaccine in the six weeks before the onset of their initial illness. Two of these patients $(6.9 \%, 95 \%$ CL $0.85 \%, 22.8 \%)$ had a recurrence of symptoms after a second, different, vaccine was subsequently administered

Of the 179 patients with CIDP, 65 had been immunised after disease onset. Five reported worsening of neurological symptoms following immunisation. In three the symptoms were similar to a typical relapse of their CIDP, but only one of these patients required treatment within two months of immunisation. The other two patients with CIDP were immunised

when already experiencing mild neurological symptoms, which then worsened, so that their modified Rankin scale score increased from 1 to 4 and they became dependent on a walking stick and unable to drive.

Of the patients with CIDP who experienced a relapse after immunisation, two relapses occurred among 23 patients who received the tetanus vaccine, giving a risk of relapse of $8.7 \%$. Two of 46 (4.3\%) patients with CIDP had relapses after influenza vaccine, of whom one had simultaneous pneumococcus vaccine. Two of six $(33 \%)$ patients, including the last mentioned, experienced relapses after pneumococcus vaccine. Fourteen patients with CIDP had no symptoms of relapse following immunisation with typhoid vaccine. Between one and seven patients with CIDP had no 
symptoms after yellow fever, diphtheria meningococcus, oral polio, $\mathrm{BCG}$, hepatitis $\mathrm{A}$, hepatitis $\mathrm{B}$, cholera, or rubella vaccine.

This audit of patients with GBS and CIDP who have received vaccines suggests that the risk of relapse following immunisation is low. The response rate to the questionnaire was small as a proportion of the membership of the GBS Support Group. This is partly because an unknown but large proportion of members are relatives or friends and not former GBS or CIDP patients.

Only 11 of 311 patients with GBS $(3.5 \%$, $95 \%$ CL $1.8 \%, 6.2 \%$ ) who had been immunised after having the disease reported a recurrence of symptoms. All of the vaccines that were associated with neurological symptom recurrence had also been received by many more patients who remained well. Some of the patients who reported symptoms after receiving vaccines had also received the same or other vaccines on other occasions without experiencing any problems. Only one respondent experienced symptoms that increased their modified Rankin scale score. The risk of relapse severe enough to alter the modified Rankin scale score is $0.3 \%$ (95\% CL $0.01 \%, 1.78 \%$ ) while the risk of a relapse requiring treatment or hospitalisation is at most $1.18 \%(95 \% \mathrm{CL})$.

It is more difficult to draw conclusions about the risk of immunisation for relapse in CIDP because our sample size was smaller. Five $(7.7 \%, 95 \%$ CL $2.5 \%, 17.0 \%)$ of 65 patients noted a return of symptoms following immunisation. The reports of minor symptoms or acceleration of deterioration following influenza and pneumococcus vaccines merit caution in recommending these immunisations in patients with CIDP, although the risk of infection in immunosuppressed patients may outweigh any potential risk. Of greatest concern is the risk of relapse following tetanus toxoid, which was $8.7 \%$ (95\% CL 1.7\%, $28.0 \%$ ) in our patient sample. In view of these figures and previous reports of relapse of CIDP following tetanus toxoid ${ }^{25}$ patients may wish to avoid routine tetanus toxoid immunisation.

Finally, it is important to acknowledge the difficulties in drawing conclusions from a questionnaire in which the patients reported their diagnostic classification and relapses. It is intuitively likely that more patients who experienced symptoms following immunisation responded to the questionnaire, which would overestimate the frequency of relapses. Consequently the true risks of relapse following immunisations after GBS or in CIDP may be less than those discovered in this audit.

\section{Acknowledgements}

We thank Mr Roland Price and members of the GBS Support Group for facilitating this audit and Dr A V Swan for statistical advice.

J Pritchard, R Mukheriee, R A C Hughes Department of Neuroimmunology, Guy's, King's \& St Thomas' School of Medicine, Hodgkin Building, Guy's Hospital, London SE 1 IUL

Competing interests: none declared

Correspondence to: Dr J Pritchard Jane.pritchard@kcl.ac.uk

\section{References}

1 da Silveira CM, Salisbury DM, de Quadros

CA. Measles vaccination and Guillain-Barré syndrome. Lancet 1997;349:14-6.

2 Hughes RA, Choudhary PP, Osborn M, et al. Immunization and risk of relapse of Guillain-Barré syndrome or chronic inflammatory demyelinating

polyradiculoneuropathy. Muscle Nerve

1996;19:1230-1.
3 Miller AE, Morgante LA, Buchwald LY, et al. A multicenter, randomized, double-blind, placebo-controlled trial of influenza immunization in multiple sclerosis. Neurology 1997;48:312-4.

4 van Swieten JC, Koudstall PJ, Visser MC, ef al. Interobserver agreement for the assessment of handicap in stroke patients. Stroke 1988;19:604-7.

5 Pollard JD, Selby G. Relapsing neuropathy due to tetanus toxoid. Report of a case. J Neurol Sci 1978;37:113-25.

\section{Hypoglycaemia induced by phenytoin treatment for partial status epilepticus}

A 22 year old woman was admitted at our epilepsy unit in status epilepticus. On examination, seizures were characterised by a confusional state with little response to externa stimuli, and recurrent, brief, tonic motor manifestations lateralised to the left side. Family history was negative for epilepsy and metabolic disorders. Full term birth was uncomplicated and first psychomotor developmental milestones were normal. In the past medical history there was no sign of any metabolic diseases. There were no reports of cognitive dysfunction or personality disturbances. At the age of 16, the patient presented with epilepsy, which was characterised by two types of seizures: global tonic seizures, which occurred yearly, and brief episodes of loss of contact without any other manifestations, which were rare. The patient was treated for many years with $20 \mathrm{mg}$ of clobazam twice daily. The awake EEGs that were performed routinely during the years of treatment with clobazam showed normal background rhythm with rare epileptiform discharges, characterised by irregular $2-3 \mathrm{~Hz}$ spike and wave complexes and localised over both frontal-central regions. Magnetic resonance imaging of the brain, which was performed at the age of 18 years, showed no abnormalities.

On the day of admission at the epilepsy unit, the patient had an urgent EEG that revealed continuous, rhythmic spikes or spike and wave complexes over both frontal-central regions with right predominance. Emergency drug treatment with intravenous lorazepam 4 mg was performed twice with a 15 minute interval, but there was no change in the clinical status. Therefore, after 30 minutes, intravenous phenytoin $1000 \mathrm{mg}$ was given by infusion over a period of 20 minutes, and then an infusion of $750 \mathrm{mg}$ of phenytoin was set up for a period of 24 hours. Clinical symptoms and EEG abnormalities rapidly improved and completely resolved after $40 \mathrm{~min}$ utes from the start of the administration of phenytoin.

Nine hours later, while the medical observation was still ongoing, the patient developed an episode of clouding of consciousness, which was preceded by prodromal symptoms, including tachycardia, sweating, ligh headedness, and irritability. On examination, there was reduction of alertness, confusion, and tachycardia. Pupils were of intermediate diameter and reactive to the light. No focal neurological signs were observed. EEG monitoring did not show any abnormalities. Emergency blood tests revealed severe hypoglycaemia ( $<20 \mathrm{mg} / \mathrm{dl})$. Prompt correction of the hypoglycaemia was obtained by the intravenous infusion of $50 \mathrm{ml}$ of $50 \%$ glucose and a consequent recovery of consciousness occurred. Phenytoin infusion was then withdrawn and oxcarbamazepine was titrated. In the following days no further episodes of hypoglycaemia were noticed. The patient was therefore investigated with the oral glucose tolerance test, which showed normal levels of plasma glucose, immunoreactive insulin, and immunoreactive insulin/plasma glucose, and with an abdominal CT scan, which did not show evidence of pancreatic insulinoma.

\section{Comment}

We have described a patient who experienced a severe episode of hypoglycaemia induced by intravenous phenytoin, which was administrated at the doses recommended for the treatment of status epilepticus. ${ }^{1}$ It is known that phenytoin interferes with carbohydrate metabolism. ${ }^{2}$ Indeed, it may inhibit the release of glucose stimulated insulin and induce a consequent hyperglycaemia. The ability of phenytoin to inhibit insulin release has been suggested to be related to the blockage of $\mathrm{Ca} 2+$ uptake via voltage dependent $\mathrm{Ca} 2+$ channels. $^{3}$ For this hyperglycaemic propriety, phenytoin has been often used in the treatment of hypoglycaemia induced by inoperable insulinomas. ${ }^{4}$

Beside the well known hyperglycaemic effect of phenytoin, it has been reported that high doses of the drug can induce hypoglycaemia. In particular, a recent study reported a case of hypoglycaemia secondary to an acute voluntary intoxication with $20 \mathrm{~g}$ of phenytoin. The authors suggested that the hypoglycaemic episode might be attributable either to an escape from the inhibitory effects of phenitoin on insulin secretion or an increased sensitivity of the tissues to insulin. ${ }^{5}$ The striking finding of our case is that the hypoglycaemia is induced by a therapeutical dose of phenytoin, and, to our knowledge, this is the first case of severe hypoglycaemia during treatment with phenytoin for status epilepticus. In this case we have indeed excluded a different aetiology of the hypoglycaemia. In particular, a possible effect on glycaemia produced by status epilepticus, ${ }^{6}$ has been considered not relevant, because the status epilepticus was partial and resolved nine hours before the onset of hypoglycaemia. However, what caused hypoglicaemia when a therapeutical dose of phenytoin was administrated is unclear, and further studies are needed to fully investigate the effects of phenytoin on charboidrate metabolism.

G Di Gennaro, P P Quarato, G B Colazza, A Mascia, F Mari, M Manfredi IRCCS "NEUROMED", Pozzilli (IS), Italy and Department of Neurological Sciences, University of Rome "La Sapienza", Rome, Italy

Correspondence to: $\operatorname{Dr} G$ Di Gennaro, IRCCS Neuromed, via Atinense, no 18, 86077, Pozzilli (IS), Italy; gdigennaro@neuromed.it

\section{References}

1 Gilliam FG. Status epilepticus. In Wyllie E, ed. The treatment of epilepsy: principles and practice. Baltimore: Williams and Wilkins, 1997:666-76

2 Mirouze J, Collard F, Thuan CD. Functional hypoglycemia: a trial of treatment with phenytoin. Effects on blood sugar and blood insulin curves. Therapie 1976:31:605-13.

3 Siegel EG, Janjic D, Wollheim CB. Phenytoin inhibition of insulin release. Studies on the involvement of $\mathrm{Ca} 2+$ fluxes in rat pancreatic islets. Diabetes 1982;31:265-9.

4 Imanaka S, Matsuda S, Ito K, et al. Medical treatment for inoperable insulinoma: clinical usefulness of diphenylhydantoin and diltiazem. Jpn J Clin Oncol 1986;16:65-71.

5 Manto M, Preiser JC, Vincent JL. Hypoglycemia associated with phenytoin intoxication. J Toxicol Clin Toxicol 1996;34:205-8. 
Table 1 Meta-analysis of $\alpha$ synuclein/non-amyloid $\beta$ component precursor allele and genotype distributions in patients with sporadic Parkinson's disease (PD) and controls in Japan

\begin{tabular}{|c|c|c|c|c|c|c|c|c|}
\hline \multirow[b]{2}{*}{ Study } & \multicolumn{6}{|c|}{ Allele* frequency } & \multicolumn{2}{|c|}{ Genotype frequency } \\
\hline & -2 & -1 & 0 & 1 & 2 & 3 & Allele $1(+)$ & Allele $1(-)$ \\
\hline \multicolumn{9}{|l|}{ Present study } \\
\hline$P D(n=165)$ & 0.009 & & 0.518 & 0.255 & 0.200 & 0.018 & 0.358 & 0.642 \\
\hline \multirow[t]{2}{*}{ Controls $(n=155)$} & 0.013 & & 0.406 & 0.355 & 0.210 & 0.016 & 0.497 & 0.503 \\
\hline & \multicolumn{6}{|c|}{$\chi^{2}=9.93, \mathrm{df}=4, \mathrm{p}=0.042, \mathrm{pc}=0.21$} & \multicolumn{2}{|c|}{$\chi^{2}=6.3, p=0.012, p c=0.072$} \\
\hline \multicolumn{9}{|l|}{ Izumi et a ${ }^{\beta}$} \\
\hline$P D(n=200)$ & & 0.003 & 0.408 & 0.248 & 0.330 & 0.013 & 0.390 & 0.610 \\
\hline \multirow[t]{2}{*}{ Controls $(n=250)$} & 0.004 & 0.002 & 0.390 & 0.320 & 0.272 & 0.012 & 0.496 & 0.504 \\
\hline & \multicolumn{6}{|c|}{$\chi^{2}=8.37, d f=5, p=0.14$} & \multicolumn{2}{|c|}{$\chi^{2}=5.05, p=0.025, p c=0.15$} \\
\hline \multicolumn{9}{|l|}{ Combined } \\
\hline$P D(n=365)$ & 0.004 & 0.001 & 0.458 & 0.251 & 0.271 & 0.015 & 0.375 & 0.625 \\
\hline \multirow[t]{2}{*}{ Controls $(n=405)$} & 0.007 & 0.001 & 0.396 & 0.333 & 0.248 & 0.014 & 0.496 & 0.504 \\
\hline & \multicolumn{6}{|c|}{$\chi^{2}=13.9, d f=5, p=0.017, p c=0.099$} & \multicolumn{2}{|c|}{$\begin{array}{l}\chi^{2}=11.4, p=0.00073, p c=0.0044 \\
\text { OR } 0.61,95 \% \mathrm{Cl} 0.45 \text { to } 0.81\end{array}$} \\
\hline
\end{tabular}

*Nomenclature of the alleles according to Xia et al. ${ }^{2}$ Alleles 1, 2, and 3 correspond to alleles 3, 2, and 1, respectively, of Krüger et al. ${ }^{4}$ pc (corrected $\mathrm{p}$ value) was obtained by multiply the $\mathrm{p}$ value by the number of alleles. $\mathrm{Cl}$, confidence interval; OR, odds ratio.

6 Shorvon S. Emergency treatment of epilepsy: acute seizures, serial seizures, seizure clusters and status epilepticus. In: Handbook of epilepsy treatment. Oxford: Blackwell Science, 2000: 173-94.

\section{Meta-analysis of $\alpha$ synuclein/ NACP polymorphism in Parkinson's disease in Japan}

$\alpha$ Synuclein is a presynaptic protein highly and broadly expressed in the brain but its normal function is unknown. ${ }^{1}$ The protein is also termed non-amyloid $\beta$ component precursor (NACP) because of its localisation in amyloid plaques of Alzheimer's disease. ${ }^{1}$ However, subsequent studies failed to confirm $\alpha$ synuclein as a component of the amyloid plaque. ${ }^{1} \alpha$ Synuclein/NACP is now known to be a major component of Lewy bodies in Parkinson's disease (PD). ${ }^{1}$ Point mutations of the $\alpha$ synuclein gene found in three independent PD families suggest that $\alpha$ synuclein may participate in the aetiology of sporadic PD. ${ }^{1}$ To address this possibility, several groups reported case-control studies using a dinucleotide repeat polymorphism in the promoter region of the gene. ${ }^{2}$ The previous Japanese study by Izumi et $a l^{3}$ found a tendency of a lower frequency of allele 1 in Japanese PD patients than in controls. ${ }^{3}$ To examine the trend of association, we performed a similar analysis in 165 PD patients and 155 healthy controls in Japan.

The patients with sporadic PD (97 women and 68 men, mean (SD) age 64 (9.6) years, mean age at onset 56 (11) years) had been under treatment at the neurological clinic of Utano National Hospital. The control group was matched for age (mean 63.0 (8.6) years), sex ratio (97 women and 58 men), and birth place (Kyoto and Osaka prefectures) with the PD patients. The controls were selected from the annual health examination at a city clinic All participants were Japanese. The institutional ethics committees approved the study protocol and informed consent was obtained from each participant. The dinucleotide repeat polymorphism was analysed as reported. ${ }^{4}$ We identified five polymerase chain reaction products with different lengths and termed them according to Xia et $\mathrm{al}^{2}$ as follows: $253 \mathrm{bp}$, allele $-2 ; 257 \mathrm{bp}$, allele 0; $259 \mathrm{bp}$, allele 1 $261 \mathrm{bp}$, allele 2; and $263 \mathrm{bp}$, allele 3. Statistical analysis was performed by $\chi^{2}$ test. The corrected $p$ value (pc) was obtained by multiplying the p value by the number of alleles.

As table 1 shows, in our study allele 1 tended to be less frequent in patients with PD than in controls $(p=0.042$ for allele distribution and $p=0.012$ for genotype distribution), although the difference was insignificant after correction by the number of alleles $(\mathrm{pc}=0.21$ for allele distribution and $\mathrm{pc}=0.072$ for genotype distribution). This result was similar to the previous Japanese work. ${ }^{3}$ To increase the power of the Japanese PD control analysis, we combined our data with those of Izumi et al. (table 1). The metaanalysis showed a significantly lower frequency of the allele 1 positive genotype in patients with PD than in controls even after correction ( $\mathrm{pc}=0.0044$, odds ratio 0.61 , $95 \%$ CI 0.45 to 0.81 ). These results suggest a negative association of allele 1 with $\mathrm{PD}$ in Japanese.

As reviewed by Farrer et al, ${ }^{5}$ results of studies of white populations have varied-some suggested a significant difference between patients with PD and controls and others did not. We did not combine Japanese data with data from white populations because of the difference in allele distribution between them: the frequencies of alleles 0,1 , and 2 in Japanese are $40 \%, 33 \%$, and $25 \%$, respectively (table 1), while the frequencies of alleles 0,1 and 2 range from $22-32 \%, 58-72 \%$, and $3-9 \%$, respectively, in white studies. ${ }^{5}$

The relation between dinucleotide repea polymorphism and the functional aspects of $\alpha$ synuclein remains unknown. Lee et $a l^{6}$ recently reported that overexpression of $\alpha$ synuclein in human neuroblastoma cell line retards cell death induced by serum withdrawal or hydrogen peroxide. This suggests that the dose of $\alpha$ synuclein may influence neuronal viability. Thus, in Japanese, allele may be associated with high expression or low degradation of $\alpha$ synuclein.

\section{Acknowledgements}

This work was supported in part by grants in aid from the Ministry of Health and Welfare of Japan (Health Science Research Grants, Research on Brain Science, and a grant in aid for Neurodegenerative Disorders).

I Mizuta, *M Nishimura, E Mizuta, S Yamasaki, **M Ohta, S Kuno Clinical Research Centre, Utano National Hospital, Kyoto 616-8255, Japan

*Also the Department of Clinical Neuroscience,

Tokushima University Hospital, Tokushima 770-8503, Japan

**Also the Department of Clinical Chemistry, Kobe Pharmaceutical University, Kobe 658-8558, Japan

Competing interests: none declared

Correspondence to: Dr S Kuno, Clinical Research Centre, Utano National Hospital, Kyoto 616-8255, Japan; yakuri@alles.or.jp

\section{References}

1 Goedert M. Alpha-synuclein and neurodegenerative diseases. Nat Rev Neurosci 2001;2:492-501.

2 Xia Y, de Silva HAR, Rosi BL, et al. Genetic studies in Alzheimer's disease with an NACP/ $\alpha$-synuclein polymorphism. Ann Neurol 1996;40:207-15.

3 Izumi $Y$, Morino H, Oda M et al. Genetic studies in Parkinson's disease with an $\alpha$-synuclein/NACP gene polymorphism in Japan. Neurosci Lett 2001;300: 125-7.

4 Krüger R, Vieira-Saecker AMM, Kuhn W, et al. Increased susceptibility to sporadic Parkinson's disease by a certain combined $\alpha$-synuclein/apolipoprotein E genotype. Ann Neurol 1999;45:611-7.

5 Farrer M, Maraganore DM, Lockhart $P$, et al. $\alpha$-Synuclein gene haplotypes are associated with Parkinson's disease. Hum Mol Genet 2001;10:1847-51.

6 Lee M, Hyun D-H, Halliwell B, et al. Effect of the overexpression of wild-type or mutant $\alpha$-synuclein on cell susceptibility to insult. J Neurochem 2001;76:998-1009. 